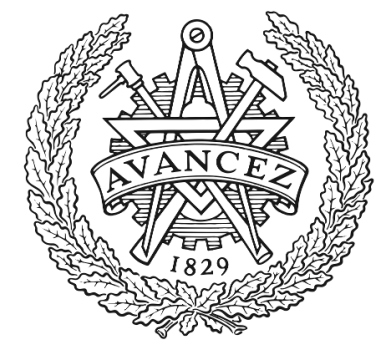

\title{
CHALMERS
}

UNIVERSITY OF TECHNOLOGY

\section{MIMO-Sparse Radars for Enhanced DOA Estimation of Spatio-Temporal Correlated Sources}

Downloaded from: https://research.chalmers.se, 2023-04-26 07:49 UTC

Citation for the original published paper (version of record):

Amani, N., Roy, V., Filippi, A. et al (2019). MIMO-Sparse Radars for Enhanced DOA Estimation of Spatio-Temporal Correlated Sources. Proceedings of the 2019 9th IEEE-APS Topical Conference on Antennas and Propagation in Wireless Communications, APWC 2019: 243-246.

http://dx.doi.org/10.1109/APWC.2019.8870432

N.B. When citing this work, cite the original published paper. 


\title{
MIMO-Sparse Radars for Enhanced DOA Estimation of Spatio-Temporal Correlated Sources
}

\author{
Navid Amani ${ }^{* \dagger}$, Venkat Roy*, Alessio Filippi*, and Rob Maaskant ${ }^{\dagger \ddagger}$ \\ *NXP Semiconductors, Eindhoven, The Netherlands \\ ${ }^{\dagger}$ Department of Electrical Engineering, Chalmers University of Technology, Gothenburg, Sweden \\ ${ }_{\ddagger}^{\ddagger}$ Electromagnetics Group, Eindhoven University of Technology (TU/e), The Netherlands \\ anavid@chalmers.se, navid.amani@nxp.com
}

\begin{abstract}
This paper investigates an enhanced direction-of-arrival (DOA) estimation problem of spatio-temporal correlated sources when a multiple-input multiple-output (MIMO) sparse radar is deployed. A virtual array with increased degrees-of-freedom (DOF) is realized within two steps by combining MIMO radar with the Khatri-Rao (KR) product approach. Since the final virtual array is a uniform linear array (ULA), a well-known spatial smoothing algorithm is applied to reinforce the rank of its covariance matrix. Meanwhile, the enhanced DOF results in a higher spatial resolution of the radar in the context of DOA estimation.
\end{abstract}

Keywords - DOA estimation, Khatri-Rao subspace, MIMO radar, sparse array.

\section{INTRODUCTION}

Autonomous cars and advanced driver assistant systems (ADASs) have attracted many research efforts, in both academia and industry, for the sake of a human safety and welfare [1]. In order to accomplish this complex mission, sensing the surrounding of vehicles by radars is one the principal backbone of the enabling technology. Direction of arrival (DOA) estimation, to spatially distinguish multiple sources and to provide an unambiguous picture of the surrounding environment, necessitates for an antenna array equipped with a signal processing unit. However, its performance highly depends on the antenna aperture size, number of elements in the array and the DOA estimation algorithm [2].

Multiple-input multiple-output (MIMO) active sensing, by deploying independent waveforms at the transmitter elements, have illustrated a superior performance by the means of a virtual array with enhanced degrees-of-freedom (DOF), in comparison with phased array radars [3]. Furthermore, an intelligent non-uniform localization of antenna elements in the aperture, e.g., minimum-redundancy arrays (MRAs) [4], co-prime arrays [5], nested arrays [6], sparse ruler arrays [7] and CRB-optimal array [8] have been developed to maximize the radar resolution and therefore the DOF by achieving more unique lags.

The concept of Khatri-Rao (KR) product along with the assumption of quasi stationary sources [9], has been shown to be capable of increasing the DOF to $2 N-1$ using an $N$-element array by vectorizing the covariance matrix of the received signal. This new vector can be treated as a single snapshot received signal at an elongated virtual array. However, the underlying simplifying assumption in their approach is the availability of sources with zero temporal correlation. In [10] and [11], it has been proved that there is an upper-bound on the value of the source cross-correlations, depending on the minimum source power, the number of sources and the angular separation among them, which it is still valid to deploy the KR approach. The feasibility of this approach has been shown in [12] by differentiating coherent sources employing the difference co-array of a sum co-array (DCSC).

In this paper, a new antenna configuration, using three transmitters and four receivers, is proposed to be used in an active radar with enhanced DOF. The idea of MIMO radar is combined with the KR product approach to produce a virtual uniform linear array (ULA) in two steps. By employing the MIMO concept, a virtual sparse ruler with eight marks is realized. Then, the DOF of the final virtual array is increased by the KR product principle. Since the final virtual array is a ULA, the spatial smoothing [13] is applied to reinforce the rank of its covariance matrix, with the cost of losing some DOFs. Afterwards, MUSIC algorithm [14] is deployed for DOA estimation. While the main concentration of the similar works is on the number of sources which can be detected, this study focuses on differentiating spatially and temporally correlated sources.

Notations: Boldface upper case and lower case denote matrices and vectors, respectively. $(.)^{*},(.)^{T}$ and $(.)^{H}$ are conjugate, transpose and Hermitian operators while $\otimes$ and $\odot$ represent the Kronecker and KR products, respectively. The notation $\operatorname{vec}($.$) stands for vectorization; that is if \mathbf{R}=$ $\left[\mathbf{r}_{1}, \ldots, \mathbf{r}_{N}\right]$ then $\operatorname{vec}(\mathbf{R})=\left[\mathbf{r}_{1}^{T}, \ldots, \mathbf{r}_{N}^{T}\right]^{T}$.

\section{DOA Estimation with Active Sensing}

First the signal model and the assumptions used in this paper are described.

\section{A. Signal Model}

Consider $K$ narrowband equi-distant sources located in the far-field of a MIMO radar which the inter-element spacing in both transmitter (TX) and receiver (RX) antennas is an integer multiple of a half-wavelength $(\lambda / 2)$. A transmitted signal by the $k$-th source is denoted by $s_{k}(t)$; then a source vector can be realized by $\mathbf{s}(t)=\left[s_{1}(t), \ldots, s_{K}(t)\right]^{T}$. Assuming $M$ and $N$ antennas at the TX and RX, respectively, an observed signal by 
an $M N$-element receive-only virtual array can be represented by $[15]$

$$
\mathbf{x}(t)=\mathbf{A s}(t)+\mathbf{n}(t), \quad t=0,1,2, \ldots
$$

where $\mathbf{A}=\mathbf{a}_{t}\left(\theta_{k}\right) \otimes \mathbf{a}_{r}\left(\theta_{k}\right) \in \mathbb{C}^{M N \times K}$ is the array manifold of the virtual array. $\mathbf{a}_{t}\left(\theta_{k}\right)$ and $\mathbf{a}_{r}\left(\theta_{k}\right)$ are the TX and RX steering vectors, respectively. Also, $\mathbf{n}(t) \in \mathbb{C}^{M N \times 1}$ represents the spatial noise satisfying $\mathbf{n} \sim \mathcal{C N}\left(\mathbf{0}, \sigma^{2} \mathbf{I}\right)$ and it is statistically independent of the source signals. Herein, $\sigma^{2}=1$ is assumed. The DOA of each source $k$ lies in the field-of-view (FOV) of the MIMO radar.

\section{B. Proposed MIMO-Sparse Radar}

In a conventional MIMO radar design, both TX and RX antennas are ULAs with uniform spacing. That is, assuming an $N$-element ULA with a unit spacing $d=\lambda / 2$ at the RX side, the inter-element spacing of an $M$-element array at the TX side is $N d$ [3]. In this case, the Kronecker product delivers an $M N$-element virtual ULA with $d$ spacing.

In order to increase the DOF further, the antenna elements at the TX and RX of a MIMO radar can be placed in such a way that the Kronecker product of their corresponding steering vectors generates a sparse ruler virtual array. A sparse ruler with length $L$ is lacking some distance marks, however, the co-difference of existing marks generates all marks from 0 to $L$ [7]. This approach of employing a difference co-array, realizable by KR product, significantly improves the DOF. The covariance matrix of the sparse ruler can be represented by

$$
\mathbf{R}_{x x}=\mathbb{E}\left\{\mathbf{x}(t) \mathbf{x}^{H}(t)\right\}=\mathbf{A} \mathbf{R}_{s s} \mathbf{A}^{H}+\mathbf{C},
$$

where $\mathbf{R}_{s s}$ denotes the source covariance matrix within which the diagonal and off-diagonal elements represent the source powers and correlation among them, respectively. $\mathbf{C} \in$ $\mathbb{C}^{M N \times M N}$ is the noise covariance matrix.

Following [9], the $\mathbf{R}_{x x}$ of an $M N$-element virtual sparse ruler array can be vectorized to form the signal snapshot steering vector of the difference co-array.

$$
\mathbf{z}=\operatorname{vec}\left(\mathbf{R}_{x x}\right) .
$$

Simplifying the model for uncorrelated sources, (3) can be rewritten as

$$
\mathbf{z}=\left(\mathbf{A}^{*} \odot \mathbf{A}\right) \mathbf{p}+\operatorname{vec}(\mathbf{C}),
$$

where $\mathbf{p}=\left[p_{1}^{2}, \ldots, p_{K}^{2}\right]^{T}$ is a vector of the source powers. Eq. (4) is not exact if the off-diagonal elements of $\mathbf{R}_{x x}$ appear to be non-zero. However, depending on the cross-correlation values the error might be tolerable [10], [11]. We investigate the effect of this error on the accuracy of the DOA estimation as the number of snapshots decreases. It should be mentioned that depending on the placement of the sensors in the MIMO configuration, the resulting sparse ruler is not necessarily minimal. Therefore, the length of the sparse ruler defines the DOF in the final difference co-array.

Since the sparse ruler with a length $L$ generates all marks from 0 to $L$, the final virtual array is a ULA. Hereupon, the spatial smoothing [13] can be applied to restore the rank of its covariance matrix, although some other works used more sophisticated DOA estimation algorithm, e.g., [12]. Afterwards, a MUSIC algorithm is applied for DOA estimation.

\section{Simulation RESULTS}

In this section, the performance of a conventional MIMO and proposed MIMO-sparse radars with $3 \mathrm{TX}, 4 \mathrm{RX}$ antennas are compared in terms of their ability to distinguish spatially and temporally correlated sources. In a conventional MIMO configuration, the RX antennas are separated by unit spacing $(d=\lambda / 2)$ and therefore the inter-element spacing at the TX side is $d=4 \times \lambda / 2=2 \lambda$. Four RX antennas are positioned at $[0,1,2,3] d$ while three TX antennas are located at $[0,4,8] d$. They are sharing the left edge element. By applying $\mathbf{A}=$ $\mathbf{a}_{t}\left(\theta_{k}\right) \otimes \mathbf{a}_{r}\left(\theta_{k}\right)$, the virtual array is a 12-element ULA with unit spacing. Therefore, using MUSIC algorithm up to eleven sources can be detected [14].

In a MIMO-sparse configuration the TX and RX elements are localized in a way that the Kronecker product of their steering vectors generates the steering vector of a sparse ruler antenna with eight marks, in order to increase the spatial resolution. Four RX antennas are located at $[0,2,7,11] d$ and three TX antennas are placed at $[0,1,11] d$. Since the aperture size of both TX and RX antennas is the same, they are sharing the left and right edge elements together. Computing $\mathbf{A}=\mathbf{a}_{t}\left(\theta_{k}\right) \otimes \mathbf{a}_{r}\left(\theta_{k}\right)$ reveals that the resulting virtual array is a sparse ruler with elements at $[0,1,2,3,8,13,18,22] d$. The sparse ruler has a length of $L=22$. Since the Kronecker product generates some unnecessary marks, e.g., $[7,11] d$, the virtual sparse ruler is not minimal.

Afterwards, the KR product concept can be applied to produce the difference co-array. Due to the sparse ruler features, the difference co-array generates all marks from $-L$ to $+L$. That is, the final virtual array is a ULA with 45 elements separated by unit spacing. However, it should be noted that the final virtual array holds a single snapshot steering vector with a rank-deficient covariance matrix. As mentioned in [6], the spatial smoothing can be applied to any array whose difference co-array is a filled ULA. Hence, this approach is utilized to restore the rank of the covariance matrix with the cost of loosing some DOFs. Upon applying the spatial smoothing at the virtual array the rank of its covariance matrix is increased up to 23. Therefore, the DOF of the MIMO-sparse configuration is still almost twice than the conventional MIMO one. Eventually, MUSIC algorithm is deployed for the DOA estimation.

As a consequence of increasing the DOF, higher spatial resolution can be achieved. Fig. 1(top), illustrates the enhanced spatial resolution of the MIMO-sparse radar in comparison with the conventional MIMO, when the angular difference between two sources is $3^{\circ}$ and sufficiently large number of snapshots is assumed. Fig. 1(middle) and (bottom) shows the configuration of the MIMO-sparse and conventional MIMO radars, respectively. The enhanced DOF is also observed in Fig. 2, when the DOA estimation of 15 sources is successfully 

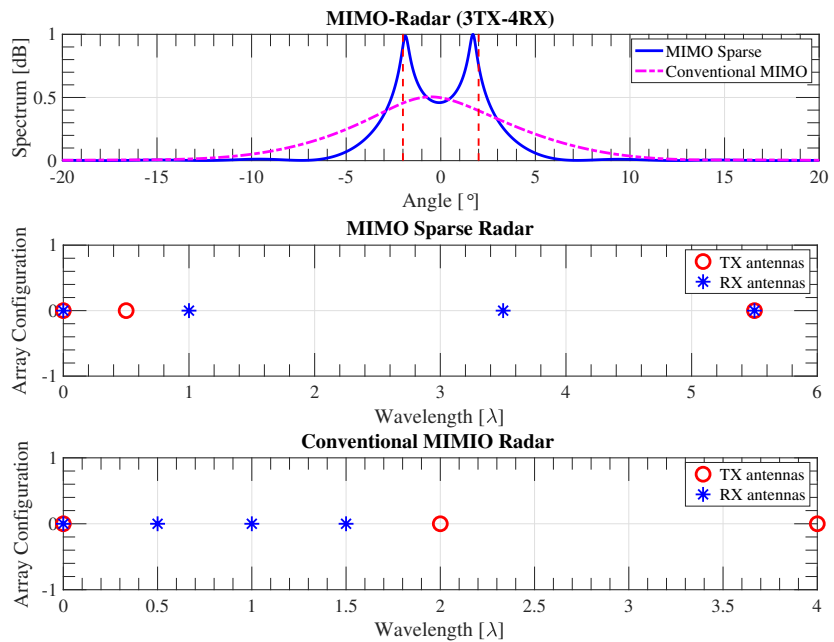

Fig. 1. (Top) Normalized spectrum of the DOA estimation using MIMO-sparse and conventional MIMO radars, (b) MIMO-sparse radar configuration, (c) Conventional MIMO configuration.

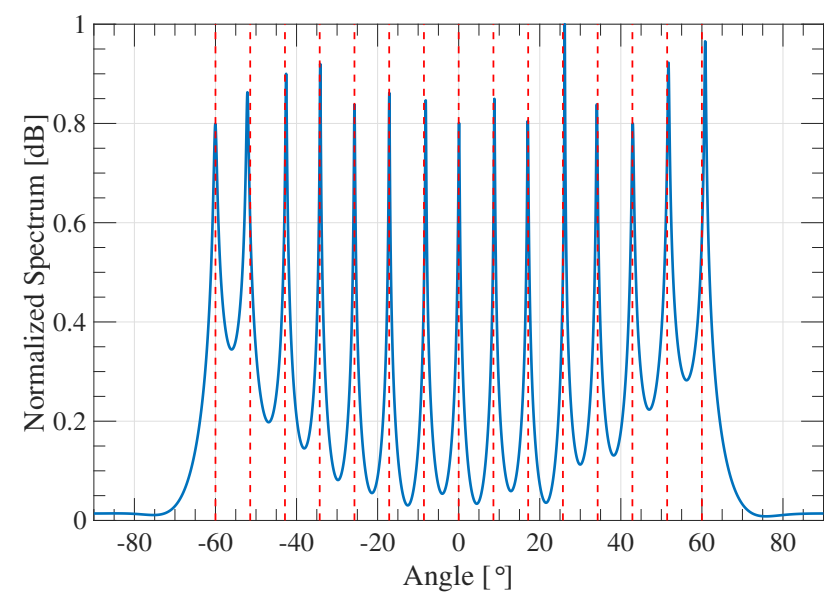

Fig. 2. DOA estimation of 15 equi-spaced sources employing a MIMO-sparse radar with 5 physical antennas. Red dashed lines represent the exact location of the sources and a blue curve shows the normalized spectrum of the DOA estimation.

accomplished using a MIMO-sparse radar with 5 physical sensors, assuming two elements are shared among TX and RX. In Fig. 2, a wide FOV of $\pm 60^{\circ}$, required in short-range automotive radars, is considered when the sources are equi-spaced in the angular domain. Also, the power of all sources is assumed to be unity. These two figures illustrate the spatial correlation reduction among sources due to the enhanced DOF.

A critical challenge in automotive radar applications is the limited number of snapshots which results in temporally correlated sources. In a worst case, having only one snapshot eventuates in coherent sources. In order to evaluate the effect of the number of snapshots on the performance of the two radar configurations, a Monte Carlo simulation is performed. It is assumed that $K$ sources are randomly distributed in the narrow FOV of $\pm 20^{\circ}$, while the minimum allowable angular separation between sources is $3^{\circ}$.

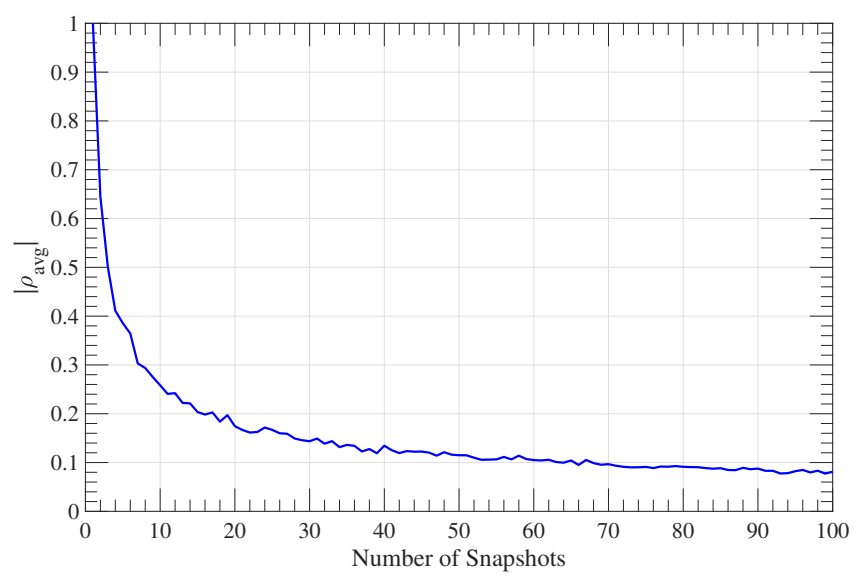

Fig. 3. Average temporal correlation among two sources versus the number of snapshots, when sources are randomly localized inside the FOV in 500 Monte Carlo simulation runs.

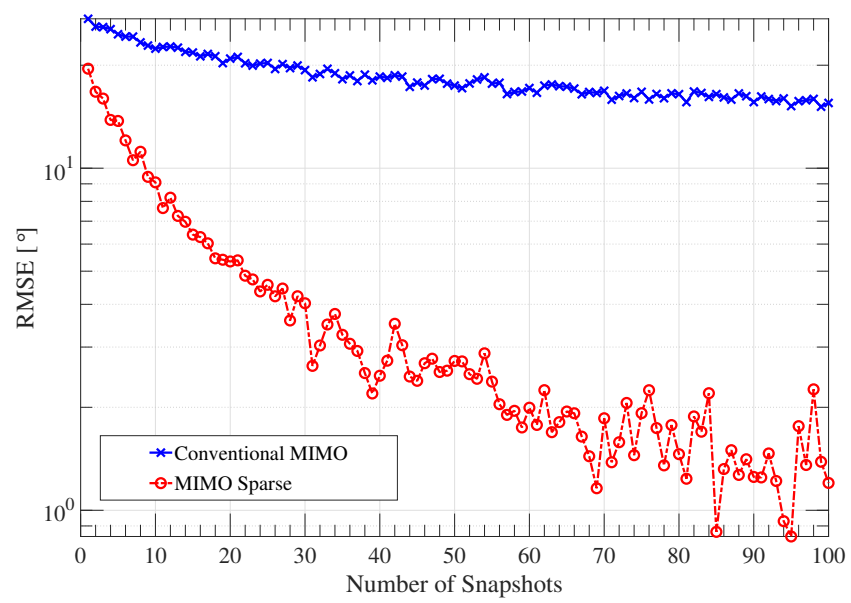

Fig. 4. RMSE versus the number of snapshots through 500 Monte Carlo simulations, when five sources are randomly localized inside the narrow FOV with minimum angular separation of $3^{\circ}$.

Such a high resolution and a limited FOV are desirable in long-range automotive radars. DOA estimation is performed, for 500 times, in a Monte Carlo simulation. Afterwards, the root mean square error (RMSE) over $N_{0}$ Monte Carlo simulation runs is computed by

$$
\mathrm{RMSE}=\sqrt{\frac{1}{N_{0} K} \sum_{i=1}^{N_{0}} \sum_{k=1}^{K}\left[\left(\theta_{k}-\hat{\theta}_{k}^{i}\right)^{2}\right]} .
$$

Fig. 3 illustrates the average correlation among two sources in the FOV. As depicted in this figure, a limited number of snapshots results in a higher temporal correlation between sources. For the single snapshot case the sources become coherent. This raises a huge error in the DOA estimation, as it is illustrated in Fig. 4.

RMSE is calculated over 500 Monte Carlo simulations for different number of snapshots in Fig. 4. In this figure, SNR $=0$ $\mathrm{dB}$ is considered. As it is shown, increasing the number of 
snapshots reduces the RMSE. Although reducing the number of snapshots degrades the DOA estimation significantly, due to the increased correlation among sources, the sparse MIMO radar performance is still better than the conventional MIMO radar, even for a single snapshot case, in terms of RMSE.

\section{CONCLUSION}

A MIMO-sparse radar has been proposed to enhance the DOA estimation performance by the means of a virtual array realizable in two steps. The increased DOF has been observed successful for spatial correlation reduction among sources. Furthermore, upon applying the spatial smoothing algorithm at the final virtual array, a superior performance has been achieved by the MIMO-sparse radar in comparison with the conventional MIMO radar in a narrow FOV, even for a single snapshot case in terms of RMSE.

\section{ACKNOWLEDGMENT}

This project has received funding from the European Union's Horizon 2020 research and innovation programme under the Marie Sklodowska-Curie grant agreement No 721732 .

\section{REFERENCES}

[1] S. M. Patole, M. Torlak, D. Wang, and M. Ali, "Automotive radars: A review of signal processing techniques," IEEE Signal Processing Magazine, vol. 34, no. 2, pp. 22-35, 2017.

[2] H. Krim and M. Viberg, "Two decades of array signal processing research: the parametric approach," IEEE signal processing magazine, vol. 13, no. 4, pp. 67-94, 1996.

[3] W. Melvin and J. Scheer, Principles of modern radar: advanced techniques. The Institution of Engineering and Technology, 2012.

[4] A. Moffet, "Minimum-redundancy linear arrays," IEEE Transactions on antennas and propagation, vol. 16, no. 2, pp. 172-175, 1968.

[5] P. P. Vaidyanathan and P. Pal, "Sparse sensing with co-prime samplers and arrays," IEEE Transactions on Signal Processing, vol. 59, no. 2, pp. 573-586, 2011.

[6] P. Pal and P. Vaidyanathan, "Nested arrays: A novel approach to array processing with enhanced degrees of freedom," IEEE Transactions on Signal Processing, vol. 58, no. 8, pp. 4167-4181, 2010.

[7] S. Shakeri, D. D. Ariananda, and G. Leus, "Direction of arrival estimation using sparse ruler array design." in SPAWC, 2012, pp. 525-529.

[8] V. Roy, S. P. Chepuri, and G. Leus, "Sparsity-enforcing sensor selection for doa estimation," in 2013 5th IEEE International Workshop on Computational Advances in Multi-Sensor Adaptive Processing (CAMSAP). IEEE, 2013, pp. 340-343.

[9] W.-K. Ma, T.-H. Hsieh, and C.-Y. Chi, "DOA estimation of quasi-stationary signals with less sensors than sources and unknown spatial noise covariance: a Khatri-Rao subspace approach," IEEE Transactions on Signal Processing, vol. 58, no. 4, pp. 2168-2180, 2010.

[10] P. Pal and P. Vaidyanathan, "Conditions for identifiability in sparse spatial spectrum sensing," in Signal Processing Conference (EUSIPCO), 2013 Proceedings of the 21st European. IEEE, 2013, pp. 1-5.

[11] A. Koochakzadeh and P. Pal, "Exact localization of correlated sources using 2D harmonics retrieval," in Signals, Systems and Computers, 2016 50th Asilomar Conference on. IEEE, 2016, pp. 1503-1507.

[12] E. BouDaher, F. Ahmad, and M. G. Amin, "Sparsity-based direction finding of coherent and uncorrelated targets using active nonuniform arrays," IEEE Signal Processing Letters, vol. 22, no. 10, pp. 1628-1632, 2015 .
[13] T.-J. Shan, M. Wax, and T. Kailath, "On spatial smoothing for direction-of-arrival estimation of coherent signals," IEEE Transactions on Acoustics, Speech, and Signal Processing, vol. 33, no. 4, pp. 806-811, 1985.

[14] R. Schmidt, "Multiple emitter location and signal parameter estimation," IEEE transactions on antennas and propagation, vol. 34, no. 3, pp. 276-280, 1986.

[15] E. Fishler, A. Haimovich, R. Blum, D. Chizhik, L. Cimini, and R. Valenzuela, "Mimo radar: An idea whose time has come," in Proceedings of the IEEE radar conference, vol. 2004. Newark, NJ, USA, 2004, pp. 71-78. 